\title{
PATRÓN DE REGENERACIÓN DE LA PLÁNTULA DE Genista monspessulana (L.) L.A.S. Johnson, EN DOS ESCENARIOS DE RESTAURACIÓN ECOLÓGICA
}

\author{
Regeneration pattern of the seedling of Genista monspessulana (L.) L.A.S. Johnson, \\ in two ecological restoration scenarios
}

Jairo Hernán Solorza-Bejarano'

Solorza-Bejarano, J.H. (2017). Patrón de regeneración de la plántula de Genista monspessulana (L.) L.A.S. Johnson, en dos escenarios de restauración ecológica. Colombia Forestal, 20(2), 131-143.

Recepción: 17 de octubre 2016

\section{Resumen}

Con el fin de mejorar los programas de control de la especie invasora Genista monspessulana, en dos escenarios de restauración ecológica con diferente disponibilidad lumínica en Bogotá, se analizó el patrón de regeneración del banco de semillas y el desarrollo inicial de los individuos. Para eso, se estudió la cobertura, abundancia, altura, longitud epigea e hipogea, número de foliolos, área foliar, diámetro de la raíz, número de ramificaciones de la raíz, presencia de nódulos y biomasa epigea e hipogea de las plántulas. El desarrollo de las plántulas, así como la regeneración del banco de semillas presentaron diferencias en los escenarios ecológicos. En el área bajo dosel arbóreo, los individuos presentaron un lento crecimiento, limitado por la cobertura de hojarasca y la baja disponibilidad lumínica, mientras que, en el área abierta con mayor incidencia lumínica, los individuos presentaron un rápido desarrollo de las hojas y raíces, además de la asociación mutualista con microorganismos que les permite mayor absorción y fijación de nitrógeno.

Palabras clave: biomasa, Bogotá, Cytisus, disponibilidad lumínica, especies invasoras, retamo liso, Teline.
Aprobación: 22 de mayo 2017

\begin{abstract}
In order to improve the control programs of the invasive species Genista monspessulana, in two scenarios of ecological restoration with different light availability in Bogotá, we analyzed the seed bank regeneration pattern and the initial development of the individuals. For that, coverage, abundance, height, epigeal and hypogeal length, the number of leaflets, leaf area, root diameter, the number of root branches, the presence of nodules and biomass epigeal and hypogeal of the seedlings were studied. Seedling development, as well as seed bank regeneration showed differences in the ecological scenarios. In the area under tree canopy, individuals presented slow growth, limited by litter cover and low light availability, while in the open area with greater light incidence, the individuals showed a rapid development of the leaves and roots, in addition to the mutualistic association with microorganisms that allows them greater absorption and fixation of nitrogen.
\end{abstract}

Keywords: biomass, Bogotá, Cytisus, light availability, invasive species, Teline.

1 Subdirección científica. Jardín Botánico de Bogotá José Celestino Mutis. Bogotá, Colombia. jsolorza@jbb.gov.co

http://dx.doi.org/10.14483/udistrital.jour.colomb.for.2017.2.a03 


\section{INTRODUCCIÓN}

Los procesos de fragmentación del paisaje, sumado a la introducción de especies exóticas, han contribuido a la generación de una dinámica de invasión que cambian las condiciones estructurales y funcionales de los ecosistemas, desviando o deteniendo los procesos de sucesión y con la consecuente pérdida de la biodiversidad y disminución en la oferta de servicios ecosistémicos (Ríos \& Vargas, 2003; Vargas \& Reyes, 2011). Estos fenómenos han despertado el interés de diferentes sectores de la sociedad que ven con urgencia la implementación de planes y programas que se orienten a la recuperación de los atributos funcionales de los ecosistemas (Villegas et al., 2013), por medio del control y facilitación de procesos biológicos a diferentes escalas.

Una de las estrategias de recuperación de los ecosistemas se aborda desde el enfoque de la restauración ecológica (Barrera \& Valdés, 2007) para facilitar el restablecimiento de atributos estructurales y funcionales, mitigar los riesgos por desastres naturales, aumentar la oferta de servicios ecosistémicos, generar procesos de conectividad con relictos de vegetación conservada y para detener y disminuir los procesos de fragmentación del paisaje (Vargas, 2007). En el caso específico de ecosistemas degradados por fenómenos de invasión por especies exóticas, se busca profundizar en el conocimiento de la biología de estas especies (Gutiérrez, 2006; Conabio, 2010) con el fin de formular las estrategias que permitan un control adecuado a corto, mediano y largo plazo, disminuyendo así el riesgo de una nueva invasión.

En Bogotá, se observa una dinámica de introducción de especies exóticas para reforestación, estabilización de suelos, cercas vivas y cultivos comerciales maderables u ornamentales (Suna Hisca, 2003; Montoya, 2004). Muchas de estas introducciones y el manejo de las mismas se hicieron sin las debidas evaluaciones sobre los riesgos de la implantación de las especies exóticas en ecosistemas que presentaban diferentes condiciones ambientales, patrones climáticos y ausencia de controladores naturales, convirtiéndolas en amenaza de invasión biológica y motor para la pérdida de biodiversidad.

El retamo liso o retamilla, Genista monspessulana (L.) L.A.S. Johnson, es una de esas especies introducidas en la ciudad de Bogotá. Este arbusto es considerado especie invasora muy agresiva (Herrera et al., 2011; Geerts et al., 2013; Cook \& Smith, 2014; Sanhueza \& Zalba, 2014). En zonas mediterráneas en Europa, de donde es originaria, las dinámicas ecológicas de sus poblaciones están asociadas a los ciclos estacionales y la ocurrencia de fenómenos de fuego (García et al., 2010). En el trópico, la especie coloniza diferentes ecosistemas, encontrándose bajo coberturas arbóreas, en áreas abiertas de talud, en claros de bosque y asociada a matrices de pastizales con suelos pobres en nutrientes o deficiencias hídricas (Bohórquez, 2013). En esos sitios G. monspessulana forma matorrales densos que incrementan el riesgo para la generación y propagación de incendios y excluyen especies nativas provocando una disminución en la biodiversidad (García et al., 2007; Pauchard et al., 2008). Esto limita la sostenibilidad de las áreas conservadas, por lo que es necesario controlar sus poblaciones para lograr los objetivos de restauración ecológica (Gutiérrez, 2006).

Para lograr un control eficiente de G. monspessulana es necesario la remoción de los individuos adultos, un continuo mantenimiento de la regeneración vegetativa y el abundante establecimiento de plántulas del banco de semillas. Esto puede variar de acuerdo al escenario alterado. La cantidad de plántulas establecidas y el mecanismo de regeneración dependerán de la intensidad y el tipo de perturbación que elimine a las plantas adultas. Por este motivo, es necesario conocer los patrones de regeneración y de desarrollo inicial de $G$. monspessulana en diferentes escenarios de alteración. Esta investigación pretende determinar el patrón de regeneración y de desarrollo inicial de los individuos de G. monspessulana en dos escenarios de restauración ecológica con diferente 
disponibilidad lumínica. Para determinar estos patrones, se analizó la respuesta morfológica adaptativa de los individuos de esta especie, así como la cantidad y distribución de biomasa en la etapa inicial del proceso de establecimiento. Adicionalmente, y con el fin de establecer estrategias eficientes de control de las plántulas de esta especie invasora, se comparó el patrón de desarrollo inicial en los dos escenarios de restauración ecológica contrastantes.

\section{MATERIALES Y MÉTODOS}

El área de estudio se encuentra en la Estación Santa Lucía (figura 1), ubicado en la localidad Rafael Uribe, de la ciudad de Bogotá. Tiene un promedio de temperatura de $13^{\circ} \mathrm{C}$, una precipitación de
$740 \mathrm{~mm}$ anuales y una humedad relativa del 58\% (SDA, 2013). El lugar se caracteriza por presentar dos zonas contrastantes en su estructura y composición florística. La primera, ubicada a $2625 \mathrm{~m}$ de altitud (04 $\left.34^{\prime} 25.3^{\prime \prime} \mathrm{N}-74^{\circ} 07^{\prime} 20.8^{\prime \prime} \mathrm{W}\right)$, es un área abierta con matorrales de G. monspessulana acompañados de escasos individuos de Ulex europaeus L. y parches de Pennisetum clandestinum Hochst. ex Chiov y Anthoxanthum odoratum L. La segunda, ubicada a una elevación de $2591 \mathrm{~m}$ de altitud $\left(04^{\circ} 34^{\prime} 28.9^{\prime \prime} \mathrm{N}-74^{\circ} 07^{\prime} 19.3^{\prime \prime}\right.$ W), es un área con árboles compuesta por especies forestales exóticas como Pinus patula Schiede ex Schltdl. \& Cham., Acacia melanoxylon R.Br., Eucalyptus globulus Labill. y Cupressus lusitanica Mill., con un sotobosque formado por matorrales de G. monspessulana.

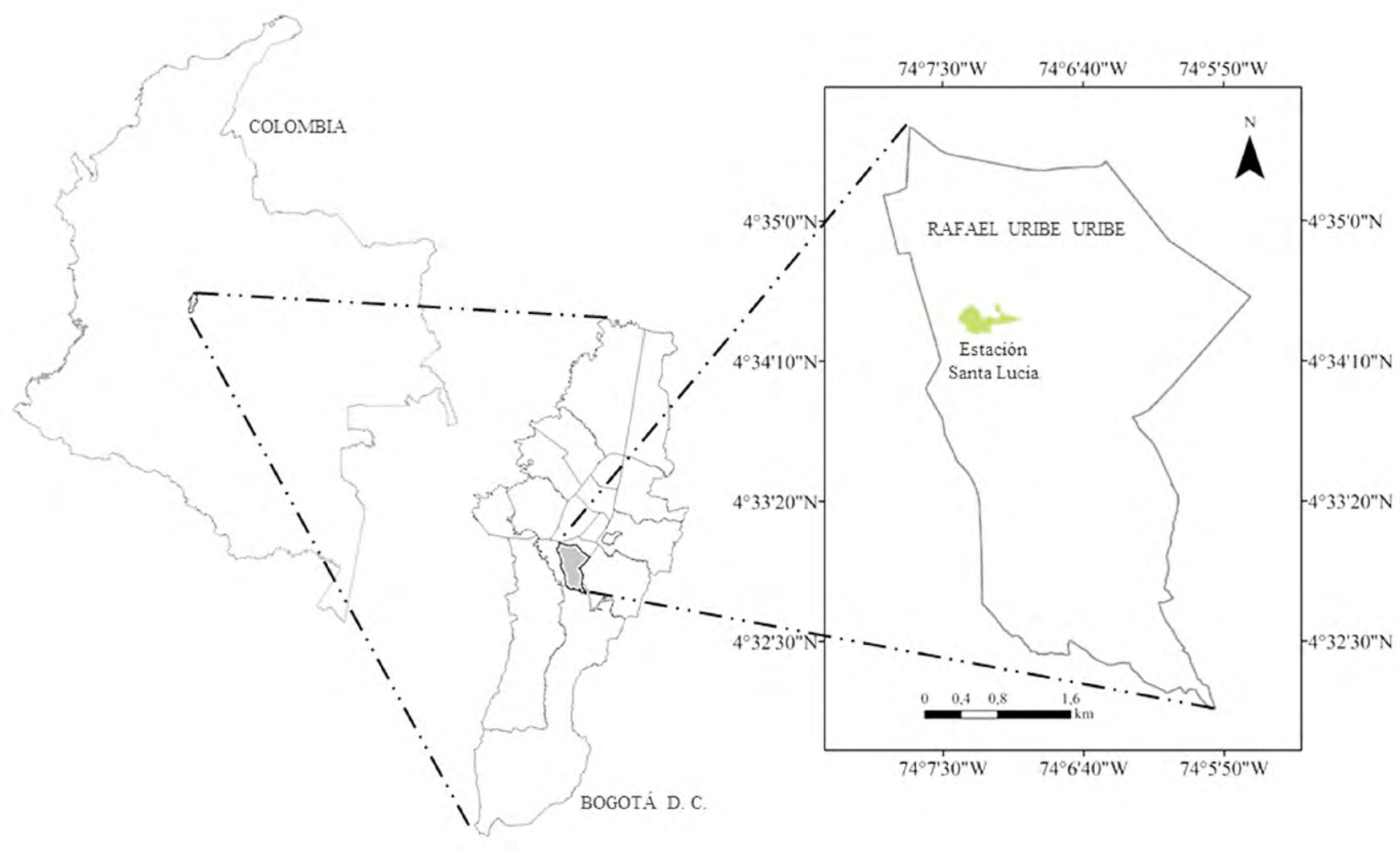

Figura 1. Localización de la Estación Santa Lucía, Bogotá, Colombia. 
Para determinar el patrón de regeneración de la plántula de G. monspessulana se estableció un método a partir del análisis estructural de la cobertura vegetal y las características morfológicas de la plántula en los primeros cuatro meses de desarrollo. Se realizó una caracterización general de las dos áreas seleccionadas, previo a la remoción de los individuos de G. monspessulana. En dos transectos de $100 \mathrm{~m}^{2}$ se midió la cobertura arbustiva de G. monspessulana y en 10 parcelas de 1 $\mathrm{m}^{2}$ distribuidos al azar se registró la cobertura herbácea y rasante, así como la presencia de plántulas de la especie invasora. Para las características morfológicas de la semilla de G. monspessulana, se tomaron cinco individuos al azar, en cada uno de ellos se removieron 20 vainas y se extrajeron las semillas para medir el diámetro ecuatorial y el diámetro polar con reglilla milimétrica bajo estereoscopio, así como el número de semillas por conteo directo.

En cada escenario de restauración ecológica se realizó la remoción total de los individuos adultos de G. monspessulana y se delimitó un cuadrante de $100 \mathrm{~m}^{2}$, dividido en cuatro subcuadrantes de 25 $\mathrm{m}^{2}$ en los que se establecieron aleatoriamente cinco parcelas de $1 \mathrm{~m}^{2}$. En cada cuadrante se tomó la medida de la incidencia lumínica a nivel del suelo, con un luxómetro digital (0-50 000 Lux). Cada mes se realizó el muestreo de las plántulas de G. monspessulana en las cinco parcelas de uno de los subcuadrantes seleccionado al azar para cada área. Previa cosecha, se midió la cobertura de plántulas de G. monspessulana mediante el establecimiento de cuadrículas de $100 \mathrm{~cm}^{2}$ al interior de la parcela, abundancia por conteo directo y altura de los individuos con calibrador pie de rey.

Todas las plántulas de G. monspesssulana en su sección epigea e hipogea cosechadas por parcela durante cuatro meses fueron procesadas en laboratorio para la medición en balanza electrónica del peso fresco y peso seco. Este último después del procesamiento en un horno de secado a $75^{\circ} \mathrm{C}$ durante 72 horas. Previo al secado, se extrajeron 10 individuos al azar de la muestra de cada parcela, a estas plántulas se les midió la longitud epigea e hipogea con calibrador pie de rey, número de foliolos por conteo directo, área foliar mediante el escaneo de los foliolos y cálculo digital, diámetro de la raíz con calibrador pie de rey, número de ramificaciones de la raíz y presencia de nódulos por conteo directo.

Se comparó cada una de las variables analizadas entre las plántulas recolectadas en cada uno de los sitios con diferente cobertura, usando análisis de variancia de una vía (ANOVA) y un $\alpha<0.05$ como probabilidad de rechazo de hipótesis nula. Antes de cada análisis se verificó la normalidad de los datos. Para el análisis estadístico se usó Excel ${ }^{\circledR}$ y el sistema básico de R (R Core Team, 2016).

\section{RESULTADOS}

Se encontró que los individuos de G. monspessulana estaban distribuidos en el área abierta con una cobertura del $65 \%$ del estrato arbustivo, acompañados de pastos de $P$. clandestinum y $A$. odoratum, con una cobertura del $30 \%$ en el estrato herbáceo. Mientras que, en el área bajo dosel arbóreo, la cobertura arbustiva de G. monspessulana fue del $15 \%$ y una cobertura de hojarasca del $70 \%$ compuesta principalmente por foliolos de acacia y acículas de pino. Para las dos áreas, la presencia de plántulas de G. monspessulana fue inferior a 15 plántulas. $\mathrm{m}^{-2}$. En las vainas de cada individuo de G. monspessulana se desarrollaron en promedio 4 \pm 1 semillas, las cuales presentaron un diámetro ecuatorial de $2.37 \pm 0.47 \mathrm{~mm}$ y diámetro polar de $2.86 \pm 0.51 \mathrm{~mm}$. La incidencia lumínica en el área abierta fue mayor de 22000 lux, mientras que en el área bajo dosel arbóreo registró entre 2000 y 4000 lux. Estos resultados de referencia permiten un contexto para el análisis de las variaciones morfológicas en la etapa inicial de desarrollo plantular de G. monspessulana (tabla 1). 
Tabla 1. Características de plántulas de G. monspessulana registradas en área abierta (AA) y bajo dosel arbóreo (DA).

\begin{tabular}{|c|c|c|c|c|c|c|c|c|c|}
\hline \multirow{3}{*}{$\begin{array}{c}\text { Unidad } \\
\text { de } \\
\text { muestreo }\end{array}$} & \multirow{3}{*}{$\begin{array}{l}\text { Variables } \\
\text { (promedio) }\end{array}$} & \multicolumn{8}{|c|}{ MES } \\
\hline & & \multirow{2}{*}{$\frac{1}{\mathrm{AA}}$} & \multicolumn{3}{|c|}{2} & \multirow{2}{*}{$\frac{3}{\mathrm{AA}}$} & \multicolumn{3}{|c|}{4} \\
\hline & & & DA & AA & DA & & DA & AA & DA \\
\hline \multirow{3}{*}{ Parcela } & $\begin{array}{l}\text { Cobertura plántulas } \\
(\%)\end{array}$ & $1.2 \pm 0.4$ & $0.7 \pm 0.3$ & $5.0 \pm 1.2$ & $1.4 \pm 0.9$ & $14.0 \pm 8.9$ & $1.4 \pm 0.5$ & $29.0 \pm 8.9$ & $1.4 \pm 0.5$ \\
\hline & $\begin{array}{l}\text { Abundancia (n. } \\
\text { individuos) }\end{array}$ & $44 \pm 24$ & $25 \pm 13$ & $169 \pm 72$ & $111 \pm 37$ & $254 \pm 144$ & $65 \pm 19$ & $824 \pm 112$ & $49 \pm 8$ \\
\hline & Altura $(\mathrm{cm})$ & $1.4 \pm 0.3$ & $2.3 \pm 0.4$ & $8.5 \pm 0.9$ & $2.5 \pm 0.1$ & $9.0 \pm 1.9$ & $6.0 \pm 0.6$ & $10.8 \pm 0.5$ & $7.6 \pm 1.0$ \\
\hline \multirow{8}{*}{ Plántula } & Foliolos (número) & $3 \pm 1$ & 2 & $19 \pm 5$ & 4 & $25 \pm 5$ & $14 \pm 3$ & $120 \pm 10$ & $27 \pm 6$ \\
\hline & Área foliar $\left(\mathrm{mm}^{2}\right)$ & $\begin{array}{l}15.72 \pm \\
7.40\end{array}$ & $\begin{array}{c}10.90 \pm \\
1.41\end{array}$ & $\begin{array}{c}276.96 \pm \\
81.02\end{array}$ & $\begin{array}{c}82.02 \pm \\
6.58\end{array}$ & $\begin{array}{l}621.16 \pm \\
125.60\end{array}$ & $\begin{array}{c}434.76 \pm \\
67.49\end{array}$ & $\begin{array}{l}3048.32 \pm \\
290.82\end{array}$ & $\begin{array}{c}890.34 \pm \\
218.49\end{array}$ \\
\hline & Longitud epigea $(\mathrm{cm})$ & $1.6 \pm 0.2$ & $1.8 \pm 0.5$ & $6.2 \pm 1.2$ & $2.5 \pm 0.2$ & $7.4 \pm 1.2$ & $5.6 \pm 0.2$ & $11.0 \pm 0.6$ & $6.7 \pm 0.2$ \\
\hline & $\begin{array}{l}\text { Longitud hipogea } \\
(\mathrm{cm})\end{array}$ & $3.3 \pm 0.7$ & $3.0 \pm 0.6$ & $5.5 \pm 1.1$ & $2.3 \pm 0.2$ & $6.4 \pm 0.3$ & $6.4 \pm 0.2$ & $8.3 \pm 0.8$ & $6.6 \pm 0.2$ \\
\hline & Longitud total $(\mathrm{cm})$ & $4.9 \pm 0.9$ & $4.8 \pm 0.8$ & $11.7 \pm 2.3$ & $4.7 \pm 0.4$ & $13.7 \pm 1.5$ & $12.0 \pm 0.4$ & $19.3 \pm 0.9$ & $13.3 \pm 0.4$ \\
\hline & $\begin{array}{l}\text { Diámetro de la raíz } \\
(\mathrm{mm})\end{array}$ & $0.24 \pm 0.08$ & $0.18 \pm 0.06$ & $1.04 \pm 0.05$ & $0.35 \pm 0.09$ & $1.44 \pm 0.28$ & $0.69 \pm 0.03$ & $1.84 \pm 0.13$ & $0.83 \pm 0.02$ \\
\hline & $\begin{array}{l}\text { Raíces secundarias } \\
\text { (número) }\end{array}$ & 0 & 0 & 1 & 0 & 2 & 0 & 3 & 0 \\
\hline & $\begin{array}{l}\text { Nódulos radiculares } \\
\text { (presencia) }\end{array}$ & No & No & Sí & No & Sí & No & Sí & No \\
\hline \multirow{3}{*}{$\begin{array}{l}\text { Biomasa } \\
\text { plántulas } \\
\text { cosecha- } \\
\text { das }\end{array}$} & Sección epigea (g.m²- & $0.2 \pm 0.1$ & 0.1 & $4.8 \pm 4.5$ & $0.4 \pm 0.3$ & $12.5 \pm 9.2$ & $0.5 \pm 0.1$ & $37.4 \pm 8.4$ & $1.0 \pm 0.3$ \\
\hline & $\begin{array}{l}\text { Sección hipogea } \\
\left(\mathrm{g} \cdot \mathrm{m}^{-2}\right)\end{array}$ & $0.1 \pm 0.1$ & 0.1 & $1.3 \pm 1.4$ & $0.2 \pm 0.1$ & $4.5 \pm 4.34$ & $0.2 \pm 0.1$ & $17.1 \pm 2.7$ & $0.4 \pm 0.2$ \\
\hline & $\begin{array}{l}\text { Total, epigea-hipogea } \\
\left(\mathrm{g} \cdot \mathrm{m}^{-2}\right)\end{array}$ & $0.3 \pm 0.3$ & 0.2 & $6.2 \pm 4.4$ & $0.6 \pm 0.3$ & $16.9 \pm 13.6$ & $0.7 \pm 0.2$ & $54.5 \pm 9.7$ & $1.4 \pm 0.5$ \\
\hline
\end{tabular}

En el área abierta se observó un cambio progresivo en las características de las plántulas de G. monspessulana regeneradas en cada periodo de estudio, con la expresión continua del banco de semillas y el aumento de la abundancia de plántulas en cada uno de los meses. De igual manera, se observó aumento en la altura, número de foliolos, área foliar, grosor y número de ramificaciones de la raíz y la presencia de nódulos (figuras 2 y 3). La inversión en biomasa en el crecimiento en la parte aérea y subterránea de la plántula varió durante los cuatro meses de desarrollo: en el primer mes ambas crecieron en una relación 1:1 (epigea: hipogea), mientras que para el cuarto mes había una relación de 2.5:1, siendo mayor en la sección epigea.
En el área bajo dosel arbóreo la regeneración de G. monspessulana fue baja y se observó un menor crecimiento en el tamaño, el número de foliolos y el área foliar. El crecimiento varió por debajo de los $0.2 \mathrm{~cm}$ en los primeros dos meses al igual que la cobertura y abundancia de plántulas. Durante el periodo de estudio se observó un incremento progresivo en la longitud de la plántula y en el diámetro de la raíz (figuras 2 y 3). No obstante, no se registró la formación de raíces secundarias, excepto un reducido número de plántulas $(n<10)$, en el cuarto mes de desarrollo.

Los individuos de G. monspessulana presentaron diferencias morfológicas en los diferentes periodos de desarrollo y entre las dos áreas evaluadas. Las principales diferencias se evidenciaron 
al cuarto mes de desarrollo en el crecimiento en altura, presencia de nódulos y mayor engrosamiento de las estructuras de soporte y anclaje en el sitio abierto comparado con el área con
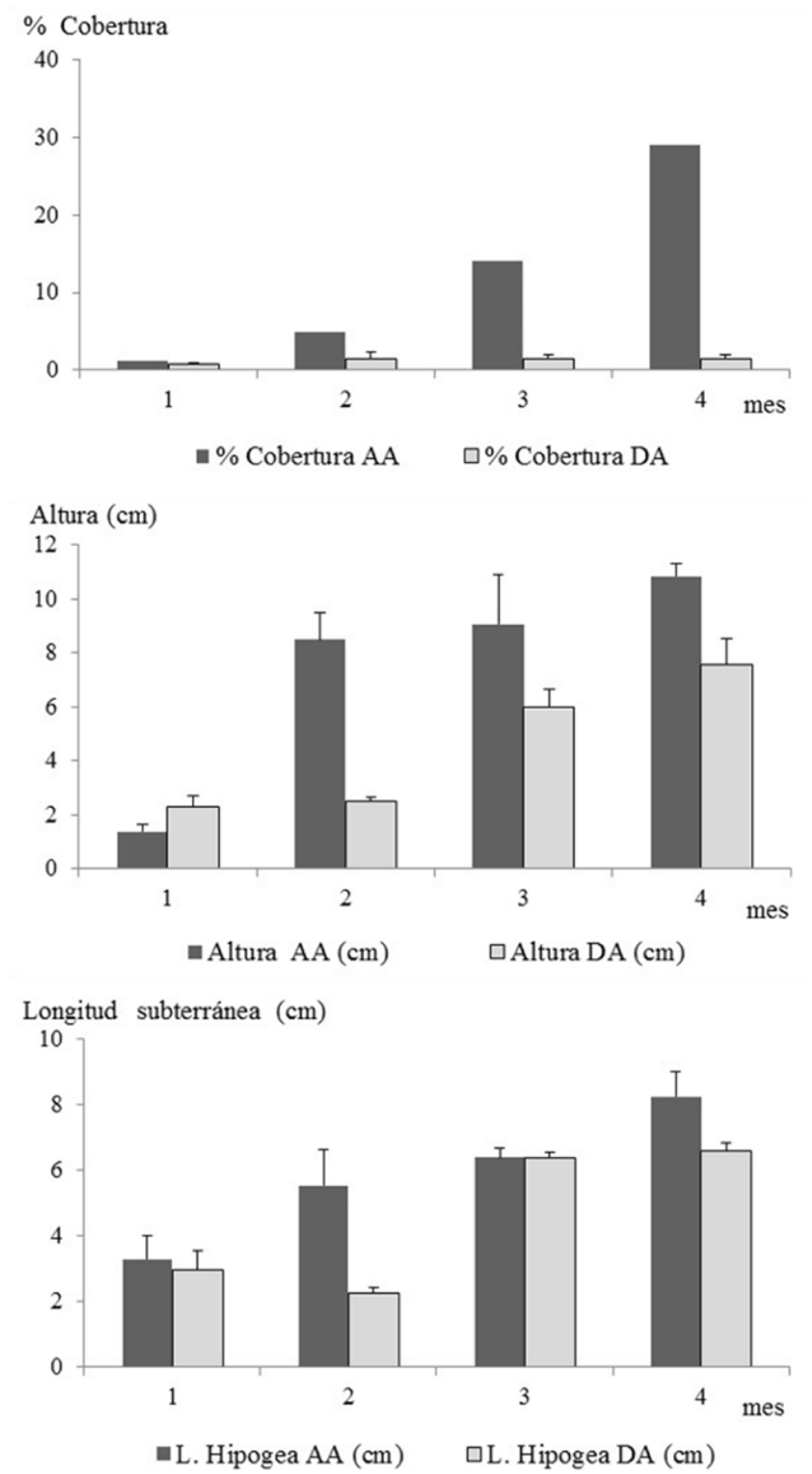

cobertura arbórea. La regeneración de individuos de G. monspessulana fue mayor en el área abierta que en el área con cobertura arbórea (tabla 2).

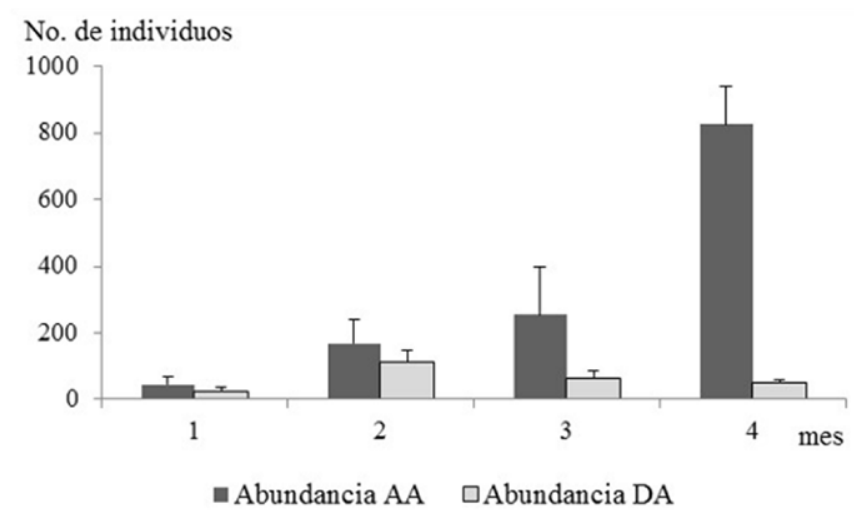

Longitud aérea $(\mathrm{cm})$

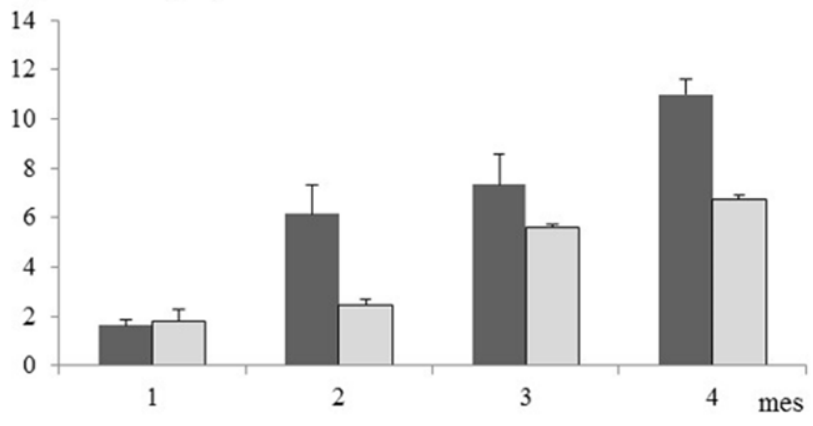

- L. Epigea AA (cm) $\quad$ L. Epigea DA $(\mathrm{cm})$

Longitud total $(\mathrm{cm})$

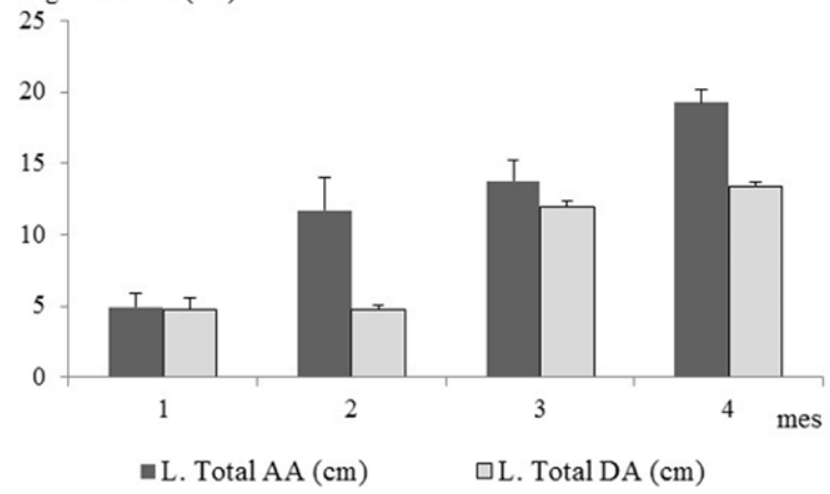

Figura 2. Variables de crecimiento de las plántulas de G. monspessulana, evaluadas en los cuatro meses de desarrollo en el área abierta (AA) y bajo dosel arbóreo (DA). 

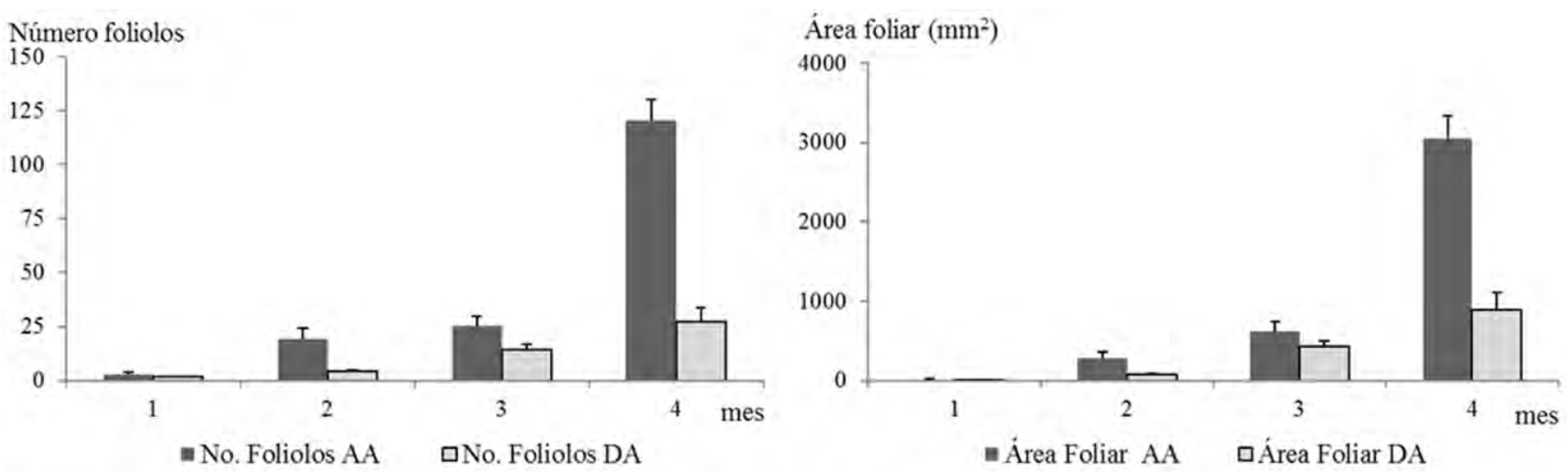

Diámetro raiz $(\mathrm{mm})$

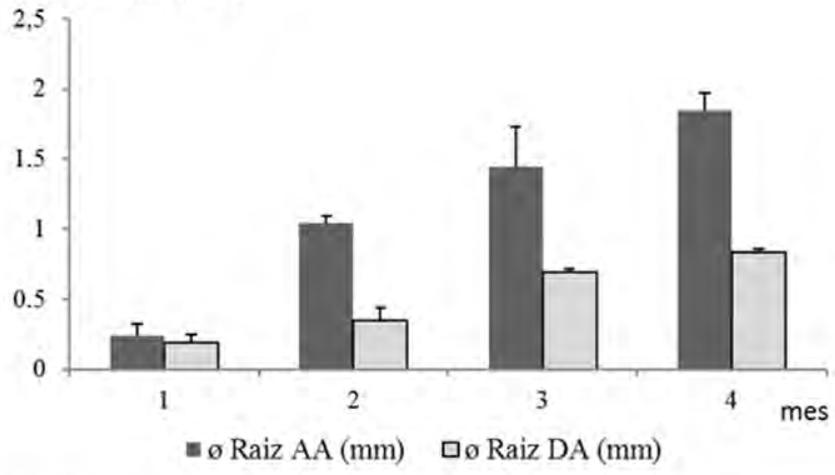

No. ramificaciones raiz
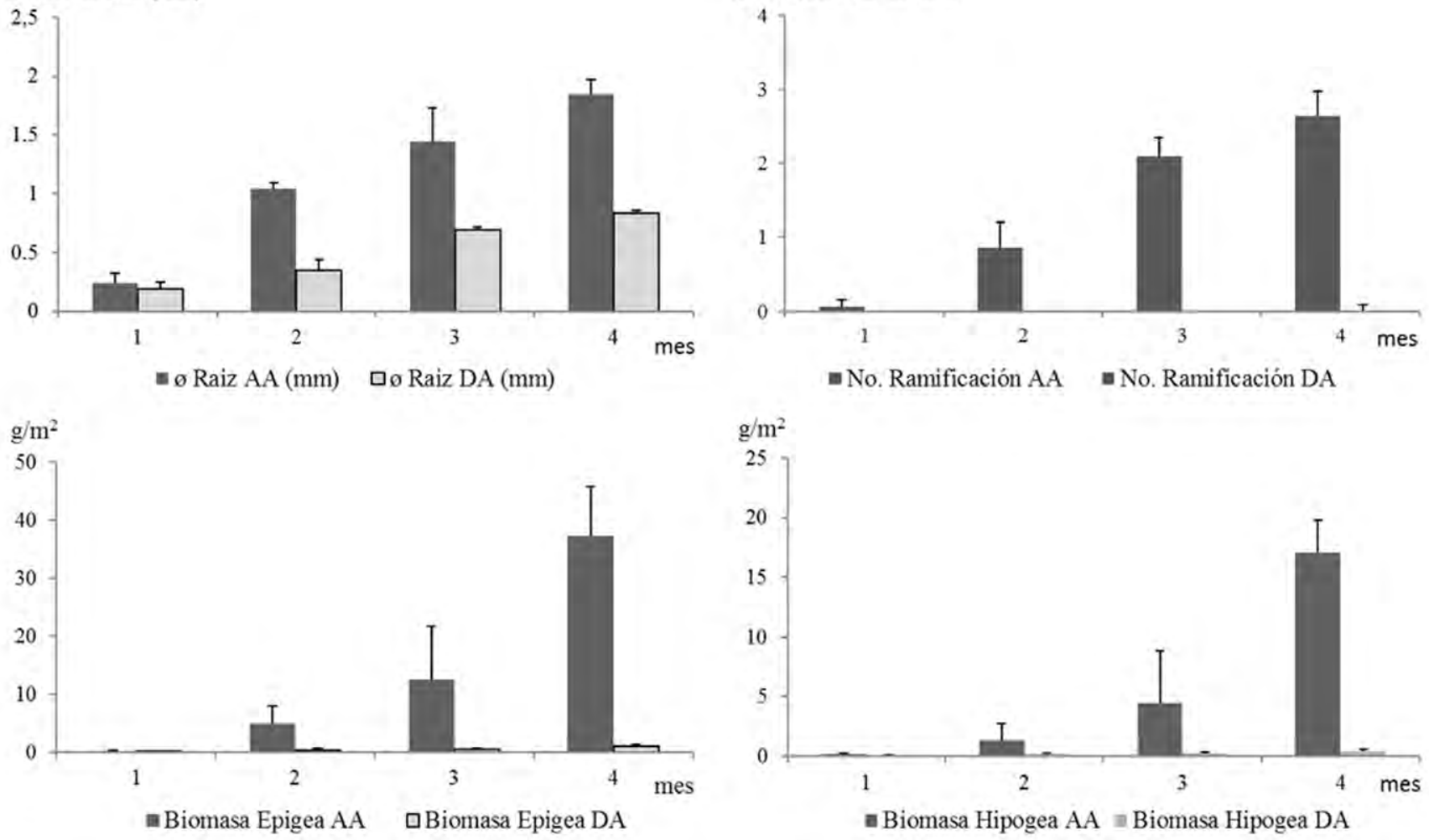

Figura 3. Variables morfológicas y biomasa de las plántulas de G. monspessulana, evaluadas en los cuatro meses de desarrollo en el área abierta (AA) y bajo dosel arbóreo (DA). 
Tabla 2. Análisis comparativo de variables morfológicas de las plántulas de G. monspessulana, registradas en área abierta (AA) y área bajo dosel arbóreo (DA).

\begin{tabular}{|c|c|c|c|c|}
\hline \multirow{2}{*}{ Variable } & \multicolumn{2}{|c|}{ Promedio } & \multirow{2}{*}{$\mathbf{F}$} & \multirow{2}{*}{$\mathbf{P}$} \\
\hline & AA & DA & & \\
\hline Cobertura $(\%)$ & $29 \%$ & $1.4 \%$ & $\mathrm{~F}_{1,8}=47.43$ & $<0.001^{* *}$ \\
\hline Abundancia (individuo) & 824 & 49 & $F_{1,8}=236.30$ & $<0.001 * *$ \\
\hline Altura $(\mathrm{cm})$ & 10.8 & 7.6 & $\mathrm{~F}_{1,98}^{1,0}=68.10$ & $<0.001^{* *}$ \\
\hline Longitud epigea $(\mathrm{cm})$ & 11 & 6.7 & $F_{1,98}=224.28$ & $<0.001^{* *}$ \\
\hline Longitud hipogea $(\mathrm{cm})$ & 8.3 & 6.6 & $\mathrm{~F}_{1,98}=35.01$ & $<0.001^{* *}$ \\
\hline Longitud total $(\mathrm{cm})$ & 19.3 & 13.3 & $\mathrm{~F}_{1,98}=163.04$ & $<0.001^{* *}$ \\
\hline Número de foliolos & 120 & 27 & $F_{1,98}=1501.42$ & $<0.001^{* *}$ \\
\hline Área foliar $\left(\mathrm{mm}^{2}\right)$ & 3048.32 & 890.34 & $\mathrm{~F}_{1,98}=1000.99$ & $<0.001^{* *}$ \\
\hline Diámetro raíz (mm) & 1.8 & 0.8 & $\mathrm{~F}_{1,98}^{1,98}=581.84$ & $<0.001^{* *}$ \\
\hline Raíces secundarias & 3 & 0 & $F_{1,98}=351.68$ & $<0.001^{* *}$ \\
\hline
\end{tabular}

**Diferencias significativas.

El desarrollo de tejidos presenta diferencias significativas entre las áreas, con una mayor producción de biomasa epigea $\left(\mathrm{F}_{1,8}=92.64 ; \mathrm{P}<0.001\right)$ y biomasa hipogea $\left(\mathrm{F}_{1,8}=192.86 ; \mathrm{P}<0.001\right)$ en el área abierta.

\section{DISCUSIÓN}

La presencia y dominio de G. monspessulana en un área degradada, con suelos poco profundos, responde a los rasgos de historia de vida que le facilitan la colonización de esos sitios. Esta especie presenta características de especies pioneras y alta capacidad de competencia con el resto de especies. En condiciones intertropicales de alta temperatura y ausencia de controladores biológicos, G. monspessulana puede establecerse en diferentes escenarios ecológicos dada su capacidad de generar un banco entre 30000 y 100000 semillas.m ${ }^{-2}$ (Sheppard, 2000) que tienen dispersión balística (obs. pers.) y resistencia y viabilidad por largos periodos de tiempo (García et al., 2007). Esta especie presenta tasas de regeneración altas, a través de renuevos vegetativos o a partir de semillas (Barrera et al., 2010), que permite la formación de un banco de semillas abundante y persistente en el tiempo a la espera de la ocurrencia de un disturbio para su activación y recolonización. Además, la capacidad de fijar nitrógeno le permite cambiar rápidamente las condiciones fisicoquímicas del suelo, lo que favorece la persistencia de la especie invasora y la exclusión de especies nativas (Quiroz, 2009).

El establecimiento de G. monspessulana corresponde a las áreas con suelos arcillosos y compactados con adición de materiales estériles y horizontes orgánicos poco desarrollados, donde predomina la colonización de especies ruderales, exóticas y el establecimiento de plantaciones forestales. Esta observación se relaciona con lo descrito por García et al. (2014), quienes registran a G. monspessulana en paisajes con fuertes disturbios en plantaciones forestales y matorrales, escenarios alterados con alta incidencia lumínica y disponibilidad de minerales en el suelo que favorecen el proceso de invasión. Esta especie oportunista genera rápidamente coberturas en el estrato rasante o herbáceo y comienzan un proceso de competencia interespecífica por los recursos y espacios, lo que provoca la homogenización de la comunidad y la exclusión de especies poco competitivas o de estadios sucesionales tardíos (Ríos \& Vargas, 2003). 
La eliminación de los individuos adultos de $G$. monspessulana y el control de las gramíneas en el área, sumado al enriquecimiento con especies nativas, permite la reactivación del proceso sucesional (Vargas et al., 2010) y el direccionamiento del mismo a la estructuración de una vegetación arbustiva y arbórea zonal. Durante el proceso sucesional en este tipo de escenarios de disturbio es común la aparición de especies oportunistas o pioneras que generan cobertura vegetal, así como cambios edáficos y microclimáticos que posteriormente permitirán el arribo de especies tardías o de lento crecimiento (Vargas, 2008), características de ecosistemas maduros. Sin embargo, en áreas disturbadas la dinámica de arribo y expresión del banco de semillas germinable facilita la presencia de especies exóticas, incluso con características invasoras (Fernández-Méndez et al. 2016; Romero et al. 2016).

El acceso a la radiación lumínica determina el patrón de regeneración y desarrollo de los individuos de G. monspessulana, producto de la expresión del banco de semillas. En el área con alta incidencia lumínica (>22 000 lux) la densidad de individuos adultos de G. monspessulana es de 2 individuos. $\mathrm{m}^{-2}$. Estos tienen una alta producción de semillas viables (Miranda, 2003), que son dispersadas hasta cuatro metros de distancia (Quiroz et al., 2009) y almacenadas en el suelo, donde la germinación es activada bajo diferentes disturbios. La expresión del banco de semillas se ve favorecida por las altas temperaturas en el suelo que activan el mecanismo de germinación (García et al., 2010) y generan hasta 900 individuos. $\mathrm{m}^{-2}$, en concordancia con lo reportado por Pauchard et al. (2008), las cuales crecen agrupadas en áreas desprovistas de vegetación herbácea, formando pequeños parches poblacionales.

El crecimiento inicial está dirigido a la apertura de los cotiledones y elongación de la raíz principal con la formación de numerosas vellosidades, aumentando el área de absorción de nutrientes y agua. En los meses siguientes la producción de tejidos de los individuos juveniles se concentra en la elongación del tallo para ganar mayor altura y obtener mayor espacio, luz y el incremento de foliolos y área foliar para aumentar la tasa fotosintética y proveer de energía al individuo. En la sección radicular se dinamiza el proceso de engrosamiento de la raíz y formación de raíces secundarias para incrementar el área de absorción y de anclaje al sustrato, facilitando el proceso de competencia y persistencia. Conforme se desarrollan las raíces secundarias, se forman nódulos radiculares producto de una asociación mutualista con cepas de Rhizhobium spp y Bradyrhizobium spp (Alarcón et al., 1997) que permiten la fijación de nitrógeno, mejorando así la capacidad de absorción de nutrientes disueltos (González, 1997).

En el área bajo dosel arbóreo la regeneración de los individuos de G. monspessulana presenta una dinámica diferente, con formación de matorrales menos densos $\left(<1\right.$ individuo. $\left.\mathrm{m}^{-2}\right)$. En este sitio las semillas dispersadas no se depositan directamente en el suelo, sino en la hojarasca, disminuyendo la densidad del banco de semillas. Por tanto, la densidad de plántulas es baja, con individuos aislados y coberturas inferiores al $1 \%$ del total del área y densidad aproximada de 110 semillas. m $^{-2}$. Esta observación se compara con lo encontrado en Chile por García et al. (2015), que registran una densidad de 319 plántulas. $\mathrm{m}^{-2}$ en un área de influencia boscosa y se complementa con lo reportado por Gómez et al. (2012), donde bajo condiciones de limitantes lumínicas se encuentra baja abundancia de individuos de G. monspessulana, en relación al banco de semillas existente. La germinación de las semillas y el crecimiento de los individuos están limitados a la baja disponibilidad lumínica que genera la cobertura arbórea y la capa de hojarasca. Se desarrollan plántulas con raíces profundas, delgadas y presencia de vellosidades, tallos elongados y curvados con cotiledones grandes. Dada la estructura poco resistente de las plántulas a los diferentes disturbios y la limitante lumínica para la producción de energía, el crecimiento epigeo e hipogeo es lento en los dos primeros meses, incrementándose desde el tercer mes de desarrollo. 
Los individuos persistentes presentan una activación del crecimiento epigeo, elongando el tallo y generando foliolos con área foliares grandes, al mismo tiempo que se incrementa la elongación de la raíz, pero sin formar raíces secundarias que le permitan aumentar la tasa de absorción de nutrientes (Lascano \& Spain, 1991) y mejoren el anclaje al sustrato. Este patrón de crecimiento está relacionado con la limitante en la producción energética que restringe la formación de tejidos nuevos. La inversión en biomasa está dirigida a la formación de las estructuras foliares con amplia área de captación lumínica para maximizar la producción energética requerida en los procesos de desarrollo. Resultados similares fueron reportados por Taboada (2013) para Genista florida, en la que observó un crecimiento equivalente de la biomasa de la raíz y la biomasa aérea de las plántulas durante los primeros tres meses.

De acuerdo al desarrollo de los individuos de G. monspessulana en las dos áreas evaluadas, se encontró que la regeneración y el crecimiento inicial es mayor en el área abierta, mientras que para el área bajo dosel arbóreo el número de individuos va decreciendo, probablemente debido a las limitadas condiciones de acceso lumínico. En cada sitio los individuos presentan una inversión en el crecimiento epigeo e hipogeo constante, con dos etapas de inactividad en los individuos que se desarrollan en el área bajo dosel arbóreo.

En las etapas iniciales de desarrollo los factores de luz, nutrientes y agua son fundamentales para el crecimiento de las plántulas (Baruch \& Fisher, 1991), siendo los altos niveles de radiación lumínica en leguminosas el factor relacionado con la sobrevivencia y el vigor de la planta (Romo, 2005; Pavón et al., 2011; Vega et al., 2011). Dado que la disponibilidad de energía por radiación lumínica en el área abierta es mayor, los individuos de G. monspessulana la orientan al incremento del área foliar y formación de mayor número de foliolos. De igual manera, hay engrosamiento de las estructuras de soporte, generando raíces secundarias y múltiples vellosidades radiculares. Este tipo de inversión no se observó en los individuos bajo el dosel arbóreo lo que los hace más susceptibles a pequeños disturbios probablemente incrementando la mortalidad.

Asociado al desarrollo morfológico, y posiblemente a las condiciones edáficas, los individuos del área abierta presentaron asociaciones mutualistas con microorganismos del suelo, evidenciable a través de la formación de nódulos. Esto les permite incrementar la tasa de absorción de nutrientes y fijación de nitrógeno, aumenta su capacidad fotosintética y, sumado a la mayor disponibilidad del recurso lumínico, contribuye a que tenga una mayor competitividad que facilita el proceso de establecimiento de la especie sobre otras especies colonizadoras.

\section{CONCLUSIONES}

El desarrollo de los individuos de G. monspessulana, presentó diferencias en la morfología en los dos diferentes escenarios ecológicos. Con mayor incidencia lumínica, los individuos de G. monspessulana muestran un rápido desarrollo de las hojas para la captación lumínica y de raíces para el soporte y anclaje al sustrato, así como la presencia de asociaciones mutualistas con microorganismos que les permite mayor absorción de nitrógeno. Bajo dosel arbóreo los individuos de G. monspessulana presentan un lento crecimiento, limitado por la cobertura de hojarasca y la baja disponibilidad lumínica necesaria para la producción de energía. Esto les impide formar estructuras para soporte, quedando susceptibles a diferentes tipos de disturbio durante la etapa inicial del desarrollo.

Estos resultados tienen implicaciones en el control de la expresión del banco de semillas de $G$. monspessulana, que requiere de estrategias de control continuas en los diferentes escenarios donde se realicen procesos de restauración ecológica, pero con mayor frecuencia e intensidad en las áreas donde haya más incidencia lumínica. 
Para las áreas bajo dosel arbóreo, las prácticas de control de expresión del banco plantular pueden hacerse en periodos entre cuatro y seis meses, ya que en su etapa inicial de desarrollo no presentan estructuras de soporte y anclaje que supongan dificultad para su remoción, además que la mortalidad de plántulas e individuos juveniles que no se adaptan a las condiciones lumínicas y de acceso a recursos es mayor. Sin embargo, se debe considerar que puede haber individuos remanentes que pueden convertirse en un nuevo foco de invasión. Por lo tanto, la duración del control plantular estará sujeto al agotamiento del banco de semillas hasta que se reduzca el riesgo de recolonización, al tiempo que se facilita el establecimiento de una cobertura vegetal nativa.

\section{AGRADECIMIENTOS}

El autor agradece al Jardín Botánico de Bogotá José Celestino Mutis y el equipo de restauración ecológica de la Subdirección Científica por la financiación y apoyo para el desarrollo del proyecto de investigación. A la bióloga Alba Luz González por su asesoría estadística y cartográfica.

\section{CONFLICTO DE INTERESES}

El autor declara no tener conflicto de intereses.

\section{CONTRIBUCIÓN POR AUTOR}

El autor único es responsable de la obra en todos los aspectos que condujeron a la elaboración de su publicación.

\section{REFERENCIAS BIBLIOGRÁFICAS}

\author{
Alarcón, E., Lozano, A. \& Chaparro, H. (1997). Carac-
} terización fenotípica de aislamientos rizobianos de acacia (Acacia sp.) y retamo (Genista monspessulana). Revista Colombiana de Química, 26(2), 21-33.

Barrera, J. \& Valdés, C. (2007). Herramientas para abordar la restauración ecológica en áreas disturbadas en Colombia. Universitas Scientiarum, 12, 12-24.

Barrera, J., Contreras, S., Garzón, N., Moreno, A. \& Montoya, S. (2010). Manual para la restauración ecológica de los ecosistemas disturbados del Distrito Capital. Bogotá: Pontificia Universidad Javeriana. $403 \mathrm{p}$.

Baruch, Z. \& Fisher, M. (1991). Factores climáticos y de competencia que afectan el desarrollo de la planta en el establecimiento de una pastura. En C. Lascano, C. \& J. Spain, Establecimiento y renovación de pasturas. Conceptos, experiencias y enfoque de la investigación. Cali (Colombia): CIAT. 426 p.

Bohórquez, C. (2013). Determinación del potencial de restauración ecológica en el Parque Nacional Enrique Olaya Herrera, II Etapa. Colombia Forestal, 16(2), 200-215.

Cook, B. S. \& Smith, L. (2014). Prerelease efficacy test of the psyllid, Arytinnis hakani, a prospective biological control agent of the invasive weed Genista monspessulana. Biocontrol Science and Technology, 24(6), 641-651.

Comisión Nacional para el Conocimiento y Uso de la Biodiversidad (Conabio). (2010). Estrategia nacional sobre especies invasoras en México. Prevención, control y erradicación. México D.F.: Offset Rebosán, S.A. de C.V. 110 p.

Fernández-Méndez, F., Velasco-Salcedo, V., Guerrero-Contecha, J., Galvis, M. \& Viana, A. (2016). Recuperación ecológica de áreas afectadas por un incendio forestal en la microcuenca Tintales (Boyacá, Colombia). Colombia Forestal, 19(2), 143-160.

García, R., Pauchard, A. \& Peña, E. (2007). Banco de semillas, regeneración y crecimiento de Genista monspessulana (L.) K. Koch después de un incendio forestal. Gayana Botánica, 64(2), 201- 210.

García, R., Pauchard, A., Cavieres, L., Peña, E. \& Rodríguez, M. (2010). El fuego favorece la invasión de Teline monspessulana (Fabaceae) al aumentar su germinación. Revista chilena de historia natural, 83(3), 443-452. 
García, R., Pauchard, A. \& Escudero, A. (2014). French broom (Teline monspessulana) invasion in south-central Chile depends on factors operating at different spatial scales. Biological invasions, 16, 113-124.

García, R., Engler, M., Peña, E. Pollnac, F. \& Pauchard, A. (2015). Fuel characteristics of the invasive shrub Teline monspessulana (L.) K. Koch. International Journal of Wildland Fire, 24, 372-379.

Geerts, S., Botha, P., Visser, V., Richardson, D. \& Wilson, J. (2013). Montpellier broom (Genista monspessulana) and Spanish broom (Spartium junceum) in South Africa: An assessment of invasiveness and options for management. South African Journal of Botany, 87, 134-145.

Gómez, P, Bustamante, R, \& San Martín, J. (2012). Estructura poblacional de Teline monspessulana (L.) K. Koch en fragmentos de bosque maulino en Chile central. Gayana Botánica, 69(1), 197-200.

González, A. (1997). Leguminosas arbustivas en sistemas agrarios sostenibles. Vida Rural, 51, 74-78.

Gutiérrez, F. (2006). Estado de conocimiento de especies invasoras. Propuesta de lineamientos para el control de los impactos. Bogotá: Instituto de Investigación de Recursos Biológicos Alexander von Humboldt, Arfo Editores e Impresores. 156 p.

Herrera, A., Carruthers, R. \& Mills, N. (2011). Introduced populations of Genista monspessulana (French broom) are more denser and produce a greater seed rain in California, USA, than native populations in the Mediterranean Basin of Europe. Biological Invasions, 13(2), 369-380.

Lascano, C. \& Spain, J. (1991). Establecimiento y renovación de pasturas. Conceptos, experiencias y enfoque de la investigación. Cali (Colombia): Ciat. $426 \mathrm{p}$.

Miranda, V. (2003). Caracterización del banco edáfico de Genista monspessulana (L.) K. Koch en tres sitios de la provincia de Concepción. (Tesis de pregrado, Facultad de Ciencias Forestales). Concepción (Chile): Universidad de Concepción. 133 p.

Montoya, S. (2004). Guía técnica para la restauración ecológica en áreas con plantaciones forestales exóticas en el Distrito Capital. Bogotá: Dama. 77 p.
Pauchard, A., García, R., Peña, E., González, C., Cavieres, L. \& Bustamante, R. (2008). Positive feedbacks between plant invasions and fire regimes: Genista monspessulana (L.) K. Koch (Fabaceae) in central Chile. Biological Invasions, 10, 547-553.

Pavón, N., Ballato-Santos, J. \& Pérez-Pérez, C. (2011). Germinación y establecimiento de Mimosa aculeaticarpa var. biuncifera (Fabaceae-Mimosoideae). Revista Mexicana de Biodiversidad, 82, 653-661.

Quiroz, C., Pauchard, A., Marticorena, A. \& Cavieres, L. (2009). Manual de plantas invasoras del centro-sur de Chile. Concepción (Chile): Universidad de Concepción. 45 p.

R Core Team. (2016). R: A language and environment for statistical computing. R Foundation for statistical Computing. Vienna. Recuperado de: https:// www.R-project.org/

Ríos, H. \& Vargas, O. (2003). Ecología de las especies invasoras. Pérez Arbelaezia, 14, 119-148.

Romero, A., Baquero, N. \& Beltrán, H. (2016). Banco de semillas en áreas disturbadas de bosque subandino en San Bernardo (Cundinamarca, Colombia). Colombia Forestal, 19(2), 181-194.

Romo, M. (2005). Efecto de la luz en el crecimiento de plántulas de Dipteryx micrantha Harms "shihuahuaco" transplantadas a sotobosque, claros y plantaciones. Ecología aplicada, 4, 1-8.

Sanhueza, C. \& Zalba, S. (2014). Banco de semillas, germinación y longevidad de semillas de retama (Spartium junceum, Fabaceae): implicancias para su control. Boletín de la Sociedad Argentina de Botánica, 49(1), 67-76.

Secretaria Distrital de Ambiente (SDA). (2013). Red de monitoreo de calidad del aire de Bogotá (RMCAB). Datos humedad relativa, precipitación y temperatura, Estación San Cristóbal. Recuperado de: http:// ambientebogota.gov.co/red-de-calidad-del-aire

Sheppard, A. (2000). Selection and testing of biological control agents for control of french broom Genista monspessulana (L.) L. Johnson. Oregon (EE.UU.): Department of Agriculture. $38 \mathrm{p}$.

Suna, H. (2003). Parque Ecológico Distrital de Montaña Entrenubes. Bogotá: Departamento Administrativo del Medio Ambiente. 473 p. 
Taboada, F., Cordine, T \& Díaz, E. (2013). Germinación y crecimiento inicial de leguminosas arbustivas de Galicia (noroeste de España). Memorias del sexto Congreso Forestal Español. Vitoria-Gasteiz: Sociedad Española de Ciencias Forestales. 12 p.

Vargas, O. (ed). (2007). Guía metodológica para la restauración ecológica del bosque altoandino. Bogotá: Universidad Nacional de Colombia. 191 p.

Vargas, O. (ed). (2008). Estrategias para la restauración ecológica del bosque altoandino. El caso de la reserva forestal municipal de Cogua, Cundinamarca. Bogotá: Universidad Nacional de Colombia. 372 p.

Vargas, O., Reyes, S., Gómez, P. \& Díaz, J. (2010). Guías técnicas para la restauración ecológica de ecosistemas. Bogotá: Universidad Nacional de Colombia. $92 \mathrm{p}$.
Vargas, O. \& Reyes S. (eds). (2011). La restauración ecológica en la práctica. Memorias del I Congreso colombiano de restauración ecológica y II Simposio nacional de experiencias en restauración ecológica. Bogotá: Universidad Nacional de Colombia. $933 \mathrm{p}$.

Vega, C., Meglioli, P. \& Villagra, P. (2011). Prosopis alpataco Phil. (Fabaceae, Mimosoideae). Kurtziana, 36(2), 53-64.

Villegas, S., Ospina, O., Escobar, G., Ramírez, W. \& Sánchez, J. (2013). Plan Nacional de Restauración. Restauración ecológica, rehabilitación y recuperación de áreas disturbadas. Bogotá: Ministerio de Ambiente y Desarrollo Sostenible. 100 p. 